TAIWANESE JOURNAL OF MATHEMATICS

Vol. 13, No. 2B, pp. 713-737, April 2009

This paper is available online at http://www.tjm.nsysu.edu.tw/

\title{
WELL-POSEDNESS FOR VECTOR QUASIEQUILIBRIA
}

\author{
Lam Quoc Anh, Phan Quoc Khanh, \\ Dang Thi My Van and Jen-Chih Yao*
}

\begin{abstract}
We consider well-posedness under perturbations of vector quasiequilibrium and bilevel-equilibrium problems. This kind of well-posedness relates Hadamard and Tikhonov well-posedness notions to sensitivity analysis and we apply largely techniques of the latter to establish sufficient conditions for wellposedness under perturbations. We also propose several new semicontinuity and quasiconvexity notions to weaken the imposed assumptions. Our results are new or include as special cases recent existing results. Many examples are provided for the illustration purpose.
\end{abstract}

\section{INTRODUCTION}

Well-posedness of optimization-related problems can be defined in two ways. The first and oldest is Hadamard well-posedness [15], which means existence, uniqueness and continuous dependence of the optimal solution and optimal value from perturbed data. The second is Tikhonov well-posedness [36], which means the existence and uniqueness of the solution and convergence of each minimizing sequence to the solution. Just after the Tikhonov's paper [36] dealing with unconstrained problems, Levitin and Polyak [26] extended the notion to constrained (scalar) optimization, allowing minimizing sequences $\left\{x_{n}\right\}$ to be outside of the feasible set $X_{0}$ and requiring $d\left(x_{n}, X_{0}\right)$ (the distance from $x_{n}$ to $\left.X_{0}\right)$ to tend to zero. Since then, various definitions of well-posedness have been proposed, extending and blending the mentioned three notions, and intensively studied. In [6] Hadamard well-posedness is extended, using Attouch-Wets topology $\tau_{a w}$ (known also as the bounded Hausdorff topology or epi-distance topology) for perturbations and convergence. It is proved in [6] that, under a constraint qualification, Tikhonov

Received November 11, 2008.

2000 Mathematics Subject Classification: 49K40, 90C31, 65K99.

Key words and phrases: Well-posedness, Unique well-posedness, Perturbations, Vector quasiequilibrium problems, Bilevel problems, Cone-quasi semicontinuity, Cone-level convexity.

*Corresponding author. 
well-posed convex programs are $\tau_{a w}$-Hadamard well-posed. Revalski [34] establishes the opposite implication, without constraint qualification. The Levitin and Polyak well-posedness is generalized in $[16,23]$ for problems with explicit constraint $g(x) \in K$, where $g$ is a continuous map between two metric spaces and $K$ is a closed set. For minimizing sequences $\left\{x_{n}\right\}$, instead of $d\left(x_{n}, X_{0}\right)$, here the distance $d\left(g\left(x_{n}\right), K\right)$ is required to tend to zero. This generalization is appropriate for penalty-type methods (e.g., penalty function methods, augmented Lagrangian methods) with iteration processes terminating when $d\left(g\left(x_{n}\right), K\right)$ is small enough (but $d\left(x_{n}, X_{0}\right)$ may be large). [13] is the first paper to characterize well-posedness by the Kuratowski measure of noncompactness of sets of approximately feasible and approximately optimal points tending to zero. This approach is developed later for various optimization-related problems in $[11,16,17]$.

Since the data of mathematical models are approximately obtained from measurements or statistical methods and since numerical algorithms for solving mathematical models produce minimizing sequences, only well-posed (in some sense) problems are significant. Fortunately, $[18,19,20]$ show that almost every usual optimization problem (e.g. quadratic problem, convex program, semi-infinite program, etc) is well-posed. Here "almost every" is in the Baire category sense or, in the Euclidean space case, means having full Lebesgue measure. For such generic well-posedness considerations, see also [21, 35]. To show also that well-posed problems are very often met, [33] proves sufficient conditions for well-posedness under lower sequential pseudocontinuity assumptions (weaker than lower sequential semicontinuity).

The condition number of a unconstrained optimization problem is the Lipschitz modulus of the unique minimizer as a vector function of the perturbing parameter. Condition number theorems (known in the literature also as distance theorems) assert that the condition number is the reciprocal of the distance of the objective function (in a unconstrained problem) to the set of ill-posed problems. Condition number theorems are investigated in connection with well-posedness in [10, 39, 40, 41].

Well-posedness for various problems related to optimization has been recently intensively considered: for variational inequalities in [7, 9, 11, 27, 31], for Nash equilibria in [29, 32], for fixed-point problems in [11, 24, 25], for inclusion problems in $[11,24,25]$ and for equilibrium problems in [22].

Well-posedness under perturbations (called also parametric well-posedness) is a blending of Hadamard and Tikhonov notions, gives also links to stability theory and seems well adapted to describe behaviors of solutions under perturbations. This issue is investigated in [22, 24, 37, 38, 41]. Well-posed bilevel problems are developed in $[12,22,28,29]$. Recently, well-posedness for vector optimization-related problems have been attracted the attention of many researchers, see e.g., $[9,17,22]$. Note that for vector problems, both minimizing sequences and well-posedness can be 
understood in many different meanings.

In this paper we consider well-posedness under perturbations for rather general vector quasiequilibrium problems. As far as we know, there is only reference [22] to deal with equilibrium (not quasiequilibrium) problems. Moreover, we investigate both problem settings: weak and strong (the latter is much less developed in the literature). We pay attention on weakening semicontinuity and generalized convexity assumptions for well-posedness. Hence in sections 2 and 4 we propose new cone semicontinuity and cone quasiconvexity notions and consider their properties for our use in establishing sufficient conditions for well-posedness in sections 3 and 5. However, we hope that these properties may be useful for other purposes as well. In section 3 we demonstrate sufficient conditions for well-posedness under perturbations for our two quasiequilibrium problems. These results are shown to include properly those of the only paper on well-posedness of equilibrium we know [22]. Since quasiequilibrium problems contain many optimization-related problems as particular cases, our results have obvious consequences for such cases. As an example, in section 5 we go in details to apply the theorems in section 3 only to wellposedness for equilibrium problems with equilibrium constraints. These problems are rather general kinds of bilevel optimization-related problems which have become increasingly attracted to researchers recently.

Our notations are usual. $R$ and $R_{+}$denote the real straight line $(-\infty,+\infty)$ and the nonnegative halfline $[0,+\infty)$, respectively. For a set $A$ in a topological space, $\operatorname{int} A$ and $\operatorname{cl} A$ stand for the interior and closure, respectively, of $A$. A cone $C$ in a topological vector space is called pointed if $C \cap(-C)=\{0\}$ and called solid if $\operatorname{int} C$ is nonempty. We will use partial orders defined by different solid convex cones in vector spaces. The following notations written for cone $C$ but may be used in the sequel for different ordering cones, since the context ensures that no confusion threatens.

$$
\begin{aligned}
& x \geq y \Leftrightarrow x-y \in C, \\
& x>y \Leftrightarrow x-y \in \operatorname{int} C, \\
& x \geq y \Leftrightarrow x-y \notin C, \\
& x \ngtr y \Leftrightarrow x-y \notin \operatorname{int} C
\end{aligned}
$$

and similarly for $\leq,<, \not \leq, \nless$.

Recall that a multimap $Q: X \rightarrow Y$ between two topological spaces is said to be upper semicontinuous (usc in short) at $x \in X$ if, for each open superset $U \supseteq Q(x)$, there is a neighborhood $N$ of $x$ such that $Q(N) \subseteq U$. $Q$ is called lower semicontinuous at $x$ if, for each open subset $U \subseteq Y$ with $Q(x) \cap U \neq \emptyset$, there is a neighborhood $N$ of $x$ such that $Q\left(x^{\prime}\right) \cap U \neq \emptyset$, for all $x^{\prime} \in N$. We also use the following two equivalent formulations for lower semicontinuity:

(a) $Q$ is lsc at $x$ if, for all $x_{\alpha} \rightarrow x$ and $y \in Q(x)$, there exists $y_{\alpha} \in Q\left(x_{\alpha}\right)$ such 
that $y_{\alpha} \rightarrow y$;

(b) $Q$ is lsc at $x$ if, for all $x_{\alpha} \rightarrow x$, one has

$$
Q(x) \subseteq \liminf Q\left(x_{\alpha}\right),
$$

where

$$
\liminf Q\left(x_{\alpha}\right):=\left\{y \in Y: \exists y_{\alpha} \in Q\left(x_{\alpha}\right), y_{\alpha} \rightarrow y\right\}
$$

\section{Quasi-SEMicontinuities of Vector Functions}

In this section we propose several kinds of quasi-semicontinuity of vector functions and investigate their properties. These notions and properties will be in use later for discussing well-posedness. Let, in this section, $X$ be a Hausdorff topological space, $Y$ be a Hausdorff topological vector space, $A \subseteq X$ be a nonempty open subset and $C \subseteq Y$ be a solid closed convex cone.

There have been several notions of semicontinuity of vector functions in the literature, but they may appear under slightly different terms. In this paper we call $h: A \rightarrow Y$ to be $C$-lower semicontinuous (shortly, $C$-lsc) at $x_{0}$ if for any neighborhood $U$ of $h\left(x_{0}\right)$, there is a neighborhood $N$ of $x_{0}$ such that $h(N) \subseteq U+C$. $h$ is called $C$-upper semicontinuous ( $C$-usc) at $x_{0}$ if for any neighborhood $U$ of $h\left(x_{0}\right)$, there is a neighborhood $N$ of $x_{0}$ such that $h(N) \subseteq U-C$.

We note the following simple relation to semicontinuity of scalar functions. Let $c \in C, f: A \rightarrow R$ and $h: A \rightarrow Y$ defined by $h(x)=f(x) c$, for $x \in A$. If $f$ is lsc (usc, respectively) at $x_{0}$, then $h$ is $C$-lsc ( $C$-usc, respectively) at $x_{0}$. Furthermore, relations to level sets are also easily obtained as follows. For $b \in Y$, we use the following notations for level sets of $h$, for different orderings $\leq$ (by the context, no confusion occurs).

$$
\begin{aligned}
& \mathrm{L}_{\leq b} h=\{x \in A: h(x) \leq b\}, \\
& \mathrm{L}_{\ngtr b} h=\{x \in A: h(x) \ngtr b\}
\end{aligned}
$$

and similarly for other level sets $\mathrm{L}_{\nless b} h, \mathrm{~L}_{\geq b} h, \mathrm{~L}_{\nless b b} h, \mathrm{~L}_{>b} h$, etc. Then it is not hard to verify the following equivalent relations.

Proposition 2.1. Let $h: A \rightarrow Y$.

(i) $h$ is $C$-lsc at $x_{0}$ if and only if $\mathrm{L}_{\ngtr b} h$ is closed at $x_{0}$ for all $b \in Y$.

(ii) $h$ is $C$-usc at $x_{0}$ if and only if $\mathrm{L}_{\nless b} h$ is closed at $x_{0}$ for all $b \in Y$.

This equivalence suggests the following natural properties weaker than $C$ semicontinuities, by imposing requirements only at a given level. Here we propose also similar definitions for other preorders $\leq$ and $\geq$. 
Definition 2.1. Let $h: A \rightarrow Y$ and $b \in Y$ be given.

(i) $h$ is called $b$-level $C$-lower semicontinuous $\left((b, C)\right.$-lsc) at $x_{0}$ if $\mathrm{L}_{\ngtr b} h$ is closed at $x_{0}$.

(ii) $h$ is called $b$-level $C$-upper semicontinuous $\left((b, C)\right.$-usc) at $x_{0}$ if $\mathrm{L}_{\nless b} h$ is closed at $x_{0}$.

(iii) $h$ is called $b$-level $C$-quasilower semicontinuous $\left((b, C)\right.$-qlsc) at $x_{0}$ if $\mathrm{L}_{\leq b} h$ is closed at $x_{0}$.

(iv) $h$ is called $b$-level $C$-quasiupper semicontinuous $\left((b, C)\right.$-qusc) at $x_{0}$ if $\mathrm{L}_{\geq b} h$ is closed at $x_{0}$.

If a statement of (i)-(iv) holds for all $b \in Y$, we omit $b$ in the corresponding terminology.

To make these definitions clearer we present equivalent statements in terms of neighborhoods as follows.

Proposition 2.2. Let $h: A \rightarrow Y$ and $b \in Y$ be fixed.

(i) $h$ is $(b, C)$-lsc at $x_{0}$ if and only if there is a neighborhood $N$ of $x_{0}$ with $h(N) \subseteq b+\operatorname{int} C$ whenever $h\left(x_{0}\right) \in b+\operatorname{int} C$.

(ii) $h$ is $(b, C)$-usc at $x_{0}$ if and only if there is a neighborhood $N$ of $x_{0}$ with $h(N) \subseteq b-\operatorname{int} C$ whenever $h\left(x_{0}\right) \in b-\operatorname{int} C$.

(iii) $h$ is $(b, C)$-qlsc at $x_{0}$ if and only if there exists a neighborhood $N$ of $x_{0}$ such that $h(N) \cap(b-C)=\emptyset$ whenever $h\left(x_{0}\right) \notin b-C$.

(iv) $h$ is $(b, C)$-qusc at $x_{0}$ if and only if there exists a neighborhood $N$ of $x_{0}$ such that $h(N) \cap(b+C)=\emptyset$ whenever $h\left(x_{0}\right) \notin b+C$.

Proof. By the similarity we verify only (iii) as an example.

"Only if". Suppose ad absurdum that $h$ is $(b, C)$-qlsc at $x_{0}$ and $h\left(x_{0}\right) \not \leq b$, but for any neighborhood $N$ of $x_{0}$, there is $x \in N$ such that, $h(x) \leq b$, i.e., there is a net $x_{\alpha} \rightarrow x_{0}$ with $h\left(x_{\alpha}\right) \leq b$. Then, as $h$ is $(b, C)$-qlsc at $x_{0}$, we get the contradiction that $h\left(x_{0}\right) \leq b$.

"If". Let $x_{\alpha} \rightarrow x_{0}$ with $h\left(x_{\alpha}\right) \leq b$. We show that $h\left(x_{0}\right) \leq b$. If $h\left(x_{0}\right) \not \leq b$, by the assumption there is a neighborhood $N$ of $x_{0}$ such that, for all $x \in N, h(x) \not \leq b$. Since $x_{\alpha} \rightarrow x_{0}$, one can assume that $x_{\alpha} \in N$, i.e., $h\left(x_{\alpha}\right) \not \leq b$, a contradiction.

The following relationships between $C$-semicontinuity and $C$ - quasisemicontinuity resemble those for the scalar counterparts, though not very clear from the above definitions and discussions.

Proposition 2.3. Let $h: A \rightarrow Y$. 
(i) If $h$ is $C$-lsc at $x_{0}$ then $h$ is $C$-qlsc at $x_{0}$.

(ii) If $h$ is $C$-usc at $x_{0}$ then $h$ is $C$-qusc at $x_{0}$.

\section{Proof.}

(i) Suppose that $h$ is $C$-1sc at $x_{0}$ and $x_{\alpha} \rightarrow x_{0}$ with $h\left(x_{\alpha}\right) \leq b$ for some $b \in Y$, but $h\left(x_{0}\right) \in Y \backslash(b-C)$. Since $h$ is $C$-lsc at $x_{0}$ and $Y \backslash(b-C)$ is open, one can assume that $h\left(x_{\alpha}\right) \in Y \backslash(b-C)+C$. So there are $t_{\alpha} \in Y \backslash(b-C)$ and $c_{\alpha} \in C$, such that $h\left(x_{\alpha}\right)=t_{\alpha}+c_{\alpha}$. As $h\left(x_{\alpha}\right) \in b-C$. There is $c_{\alpha}^{\prime} \in C$, such that $h\left(x_{\alpha}\right)=b-c_{\alpha}^{\prime}$. Therefore, $t_{\alpha}=b-c_{\alpha}^{\prime}-c_{\alpha} \in b-C$, which is impossible as $t_{\alpha} \in Y \backslash(b-C)$.

(ii) It may be checked similarly.

The following example shows that the converse of Proposition 2.3 is not true.

Example 2.1. Let $X=R, Y=R^{2}, A=R, C=R_{+}^{2}, x_{0}=0$ and

$$
h(x)= \begin{cases}(x, 1) & \text { if } x \leq 0, \\ \left(-\frac{1}{x}, \frac{1}{x}\right) & \text { otherwise. }\end{cases}
$$

Then $h$ is both $C$-qlsc and $C$-qusc at 0 . Indeed, by the similarity we check only the latter property. For any sequence $x_{n} \rightarrow 0$ such that $h\left(x_{n}\right) \geq b$ for some $b \in R$ and all $n$, there is a subsequence $x_{n_{k}}$ such that $x_{n_{k}} \leq 0$, for all $n_{k}$, (if for all subsequences $x_{n_{k}}, x_{n_{k}}>0$, since $x_{n_{k}} \rightarrow 0$, one has $\left.h\left(x_{n_{k}}\right) \rightarrow(-\infty,+\infty) \geq b\right)$. $h\left(x_{n_{k}}\right) \geq b$ means that $x_{n_{k}} \geq b_{1}$ and $1 \geq b_{2}$, and hence $0 \geq b_{1}$. Therefore, $h\left(x_{0}\right)=(0,1) \geq b$, i.e., $h$ is $(b, C)$-qusc at 0 . As $b$ is arbitrary, $h$ is $C$-qusc at 0 .

However, $h$ is neither $C$-lsc nor $C$-usc at 0 . Indeed, we check only the latter. Let $b=(2,2)$ and $x_{n}=\frac{1}{n}$. Then $h\left(x_{n}\right) \nless b$ for large $n$. But $h(0)=(0,1)<b$. Consequently, $h$ is not $(b, C)$-usc at 0 .

The relations asserted in Proposition 2.3 cannot be similarly stated for a particular level $b$ (unlike the scalar case) as ensured by the example below.

Example 2.2. Let $X, Y, A, C$ and $x_{0}=0$ be as in Example 2.1 and

$$
h(x)= \begin{cases}(-1,1) & \text { if } x=0 \\ (-1,-1) & \text { otherwise }\end{cases}
$$

Then, for all $x \in R, h(x) \ngtr 0$ and hence $h$ is $(0, C)$-lsc at 0 , since $\mathrm{L}_{\ngtr 0} h$ is closed. But $h$ is not $(0, C)$-qlsc at 0 , since $\mathrm{L}_{\leq 0} h$ is not closed. Indeed, let $x_{n}=\frac{1}{n} \rightarrow 0$. Then $h\left(x_{n}\right)=(-1,-1) \leq 0$, but $h(0)=(-1,1) \not \leq 0$. So $\mathrm{L}_{\leq 0} h$ is not closed. 
Now we modify $h$ as

$$
h(x)= \begin{cases}(-1,1) & \text { if } \quad x=0, \\ (1,1) & \text { otherwise. }\end{cases}
$$

Then, for all $x \in R, h(x) \nless 0$, whence $h$ is $(0, C)$-usc at 0 , since $\mathrm{L}_{\nless 0} h$ is closed. But $h$ is not $(0, C)$-qusc at 0 , since $\mathrm{L}_{\geq 0} h$ is not closed. Indeed, let $x_{n}=\frac{1}{n}$. Then $h\left(x_{n}\right)=(1,1) \geq 0$, but $h(0)=(-1,1) \nsupseteq 0$. Hence, $\mathrm{L}_{\geq 0} h$ is not closed.

For our well-posedness consideration in sections 3 and 4 we need to develop sum rules for generalized semicontinuous and quasi-semicontinuous functions.

Proposition 2.4. Let $X, Z$ be topological spaces, $A \subseteq X$ and $B \subseteq Z$ be nonempty open sets, $C \subseteq Y$ be a solid closed convex cone and $f: A \rightarrow Y, g$ : $B \rightarrow Y, A \subseteq X, B \subseteq Z$. If $f$ and $g$ are $C$-lsc (or $C$-usc) at $x_{0}$ and $y_{0}$, respectively, then $h=f+g$ is $C$-lsc (or $C$-usc) at $\left(x_{0}, y_{0}\right)$.

Proof. By the similarity and symmetry we demonstrate only for the $C$-upper semicontinuity. Let $U$ be any open superset of $f\left(x_{0}\right)+g\left(y_{0}\right)$. There are open neighborhoods $U_{1}$ and $U_{2}$ of $f\left(x_{0}\right)$ and $g\left(x_{0}\right)$, respectively, such that $U_{1}+U_{2} \subseteq U$. Since $f$ and $g$ are $C$-lsc, there are open neighborhoods $N_{1}$ and $N_{2}$ of $x_{0}$ and $y_{0}$, respectively, such that $f(x) \in U_{1}-C$ and $g(y) \in U_{2}-C$, for all $(x, y) \in N_{1} \times N_{2}$. Hence $h(x, y)=f(x)+g(y) \in U_{1}+U_{2}-C \subseteq U-C$, for all $(x, y) \in N_{1} \times N_{2}$, i.e., $h$ is $C$-usc at $\left(x_{0}, y_{0}\right)$.

Unfortunately, Proposition 2.4 cannot be extended for $C$-quasi semicontinuous functions as shown by the following example.

Example 2.3. Let $X, Y, A, C$ and $x_{0}$ be as in Example 2.1. Let $Z=B=X$, $y_{0}=x_{0}$ and $f$ be defined as $h$ in Example 2.1. Let

$$
g(x)= \begin{cases}(x, 1) & \text { if } x \leq 0 \\ \left(\frac{1}{x},-\frac{1}{x}\right) & \text { otherwise }\end{cases}
$$

By similar arguments as those of Example 2.1, we see that $f$ and $g$ are $C$-qlsc at 0. Furthermore,

$$
(f+g)(x)= \begin{cases}(2 x, 2) & \text { if } x \leq 0 \\ (0,0) & \text { otherwise }\end{cases}
$$

For $x_{n}=\frac{1}{n} \rightarrow 0$, one has $(f+g)\left(x_{n}\right)=(0,0) \leq(1,1)$, but $(f+g)(0)=(0,2) \not \leq$ $(1,1)$. So $f+g$ is not $C$-qlsc at 0 . 
Let us now modify $f$ and $g$ by setting

$$
\begin{aligned}
& f(x)= \begin{cases}(x,-1) & \text { if } x \leq 0, \\
\left(-\frac{1}{x}, \frac{1}{x}\right) & \text { otherwise }\end{cases} \\
& g(x)= \begin{cases}(x,-1) & \text { if } x \leq 0, \\
\left(\frac{1}{x},-\frac{1}{x}\right) & \text { otherwise. }\end{cases}
\end{aligned}
$$

Then $f$ and $g$ are $C$-qusc at 0 . But

$$
(f+g)(x)= \begin{cases}(2 x,-2) & \text { if } x \leq 0 \\ (0,0) & \text { otherwise }\end{cases}
$$

is not $C$-qusc at 0 because, for $x_{n}=\frac{1}{n} \rightarrow 0$, we have $(f+g)\left(x_{n}\right)=(0,0) \geq$ $(-1,-1)$, but $(f+g)(0)=(0,-2) \nsupseteq(-1,-1)$. 2.4 .

Proposition 2.5. Assume that $X, Y, Z, A, B, C, f$ and $g$ are as in Proposition

(i) If $f$ is $C$-qlsc at $x_{0}$ and $g$ is $C$-lsc at $y_{0}$, then $h=f+g$ is $C$-qlsc at $\left(x_{0}, y_{0}\right)$.

(ii) If $f$ is $C$-qusc at $x_{0}$ and $g$ is $C$-usc at $y_{0}$, then $h=f+g$ is $C$-qusc at $\left(x_{0}, y_{0}\right)$.

Proof. By the similarity and symmetry we demonstrate only (i). For arbitrary $b \in Y$, assume that $\left(x_{\alpha}, y_{\alpha}\right) \rightarrow\left(x_{0}, y_{0}\right)$ and $h\left(x_{\alpha}, y_{\alpha}\right) \leq b$. For each $e \in \operatorname{int} C$, as $g$ is $C$-lsc at $y_{0}$, there is a subnet $y_{\beta}$ such that $g\left(y_{\beta}\right)>g\left(y_{0}\right)-e$, for all $\beta$. Hence

$$
f\left(x_{\beta}\right)+g\left(y_{0}\right)-e \leq b .
$$

As $f$ is $C$-qlsc at $x_{0}$,

$$
f\left(x_{0}\right)+g\left(y_{0}\right)-e \leq b,
$$

Consequently, $f\left(x_{0}\right)+g\left(y_{0}\right) \leq b$. Thus, $h$ is $(b, C)$-qlsc at $\left(x_{0}, y_{0}\right)$. By the arbitrariness of $b, h$ is $C$-qlsc at $\left(x_{0}, y_{0}\right)$.

The following examples prevent us to state results similar to Prepositions 2.4 and 2.5 for a given fixed level $b$.

Example 2.4. Let $A=R, Y=R^{2}, C=R_{+}^{2}, x_{0}=0$ and $f, g: R \rightarrow R^{2}$ be defined by $g(x)=(2,-1)$ for all $x$, and

$$
f(x)= \begin{cases}(-1,2) & \text { if } \quad x=0 \\ (-3,-3) & \text { otherwise }\end{cases}
$$


Then $f$ is $(0, C)$-lsc at 0 , since $\mathrm{L}_{\ngtr 0} f$ is closed. $g$ is continuous, whence $(0, C)$-lsc at 0 . But

$$
(f+g)(x)= \begin{cases}(1,1) & \text { if } x=0 \\ (-1,-4) & \text { otherwise }\end{cases}
$$

is not $(0, C)$-1sc at 0 , since $\mathrm{L}_{\ngtr 0} h$ is not closed. Indeed, let $x_{n}=\frac{1}{n}$. Then $h\left(x_{n}\right)=$ $(-1,-4) \ngtr 0$, but $h(0)=(1,1)>0$. So $\mathrm{L}_{\ngtr 0} h$ is not closed. Therefore, from the fact that $f$ is $\left(b_{1}, C\right)$-lsc at $x_{0}$ and $g$ is $\left(b_{2}, C\right)$ lsc at $x_{0}$, we cannot deduce that $f+g$ is $\left(b_{1}+b_{2}, C\right)$-1sc at $x_{0}$. If $b_{1}=b_{2}, f$ is $(b, C)$-lsc at $x_{0}$ and $g$ is even continuous at $x_{0}$, we do not have that $f+g$ is $(b, C)$-lsc at $x_{0}$.

To get an counterexample for $(b, C)$-upper semicontinuity we modify $f$ and $g$ as follows. Put $g(x)=(1,-2)$ and

$$
f(x)= \begin{cases}(-2,1) & \text { if } \quad x=0 \\ (3,3) & \text { otherwise. }\end{cases}
$$

Then $f$ is $(0, C)$-usc at 0 and $g$ is continuous (and hence $(0, C)$-usc) at 0 . But

$$
(f+g)(x)= \begin{cases}(-1,-1) & \text { if } x=0 \\ (4,1) & \text { otherwise }\end{cases}
$$

is not $(0, C)$-usc at 0 , since $\mathrm{L}_{\nless 0} h$ is not closed. Indeed, let $x_{n}=\frac{1}{n} \rightarrow 0$. Then $h\left(x_{n}\right)=(4,1) \nless 0$, but $h(0)=(-1,-1)<0$, whence $\mathrm{L}_{\nless 0} h$ is not closed. This example shows that from $f$ being $(b, C)$-usc and $g$ being even continuous at $x_{0}$, it does not follow that $f+g$ is $(b, C)$-usc at $x_{0}$,

Passing to discussions for $C$-quasi semicontinuity we have similar counterexamples as follows.

Example 2.5. Let $Y, A, C$ and $x_{0}=0$ be as in Example 2.4. Let $f, g: R \rightarrow R^{2}$ be defined by $g(x)=(2,2)$ for all $x$, and

$$
f(x)= \begin{cases}(-1,-1) & \text { if } \quad x=0, \\ (-3,-3) & \text { otherwise. }\end{cases}
$$

Then $f$ is $(0, C)$-qlsc and $g$ is continuous (and hence $(0, C)$-lsc) at 0 . But

$$
(f+g)(x)= \begin{cases}(1,1) & \text { if } x=0 \\ (-1,-1) & \text { otherwise }\end{cases}
$$

is not $(0, C)$-qlsc at 0 (to see this let $x_{n}=\frac{1}{n}$ ). 
A similar assertion for $(b, C)$ - quasi upper semicontinuity is seen by setting $g(x)=(-2,-2)$ and

$$
f(x)= \begin{cases}(1,1) & \text { if } x=0, \\ (3,3) & \text { otherwise }\end{cases}
$$

The following example indicates that, for a fixed level $b$, the sum of a continuous function and a $(b, C)$-lsc function may not be $(b, C)$-qlsc.

Example 2.6. Let us modify from the previous example only $f(x)=(2,2)$ and

$$
g(x)= \begin{cases}(-1,-1) & \text { if } x=0, \\ (-3,-3) & \text { otherwise }\end{cases}
$$

Then, $f$ is continuous and $g$ is $(0, C)$-lsc at 0 . But

$$
(f+g)(x)=\left\{\begin{array}{lr}
(1,1) & \text { if } x=0 \\
(-1,-1) & \text { otherwise }
\end{array}\right.
$$

is not $(0, C)$-qlsc at 0 .

Now let $f(x)=(-2,-2), x_{0}=0$ and

$$
g(x)= \begin{cases}(1,1) & \text { if } \quad x=0 \\ (3,3) & \text { otherwise }\end{cases}
$$

Then $f$ is continuous and $g$ is $(0, C)$-usc at 0 . But

$$
(f+g)(x)= \begin{cases}(-1,-1) & \text { if } x=0 \\ (1,1) & \text { otherwise }\end{cases}
$$

is not $(0, C)$-qusc at 0 .

\section{Well-POSEDNESS FOR QUASIEQUILIBRIUM Problems}

We are concerned with the following quasiequilibrium problems, which are perturbed. Let the decision space $X$ be a Hausdorff topological space and the range space $Y$ be a Hausdorff topological vector space. Let $A \subseteq X$ be a nonempty set and the ordering cone $C \subseteq Y$ be nonempty convex, closed, pointed and solid. Let the constrained sets be depending on $x \in X$ and defined by $K_{i}: A \rightarrow 2^{A}, i=1,2$. 
Let the machinery of the problems be expressed by $f: A \times A \rightarrow Y$. The following two quasiequilibrium problems are concerned

(WQEP) Find $\bar{x} \in K_{1}(\bar{x})$ such that, for all $y \in K_{2}(\bar{x})$,

$$
f(\bar{x}, y) \nless 0 ;
$$

(SQEP) Find $\bar{x} \in K_{1}(\bar{x})$ such that, for all $y \in K_{2}(\bar{x})$,

$$
f(\bar{x}, y) \geq 0 \text {. }
$$

The notations (WQEP) and (SQEP) mean "Weak Quasiequilibrium Problem" and "Strong Quasiequilibrium Problem", respectively. The solution sets of (WQEP) and (SQEP) are denoted by $S^{w}$ and $S^{s}$, respectively.

Now assume that these problems suffer perturbations, which are expressed in terms of a perturbing parameter $\lambda$ in a Hausdorff topological space $\Lambda$. This means that our problems are embedded into the following families, for $\lambda \in \Lambda$,

$\left(\mathrm{WQEP}_{\lambda}\right)$ Find $\bar{x} \in K_{1}(\bar{x}, \lambda)$ such that, for all $y \in K_{2}(\bar{x}, \lambda)$,

$$
f(\bar{x}, y, \lambda) \nless 0 ;
$$

$\left(\operatorname{SQEP}_{\lambda}\right)$ Find $\bar{x} \in K_{1}(\bar{x}, \lambda)$ such that, for all $y \in K_{2}(\bar{x}, \lambda)$,

$$
f(\bar{x}, y, \lambda) \geq 0 \text {. }
$$

Assume that the original problems are corresponding to $\lambda=\bar{\lambda}$, i.e., (WQEP) $=\left(\mathrm{WQEP}_{\bar{\lambda}}\right)$ and $(\mathrm{SQEP})=\left(\mathrm{SQEP}_{\bar{\lambda}}\right)$. We adapt the notions of asymptotically minimizing sequences and well-posedness under perturbations introduced by Zolezzi for unconstrained optimization in [37] and developed for various problem settings in $[22,25,38,41]$ to our problems as follows.

Definition 3.1. Let $\left\{\lambda_{\alpha}\right\} \subseteq \Lambda$ be a net converging to $\bar{\lambda}$. A net $\left\{x_{\alpha}\right\} \subseteq$ $K_{1}\left(x_{\alpha}, \lambda_{\alpha}\right)$ is said to be an asymptotically solving net for (WQEP) ((SQEP), respectively) corresponding to $\left\{\lambda_{\alpha}\right\}$ if there exists a net $\left\{e_{\alpha}\right\} \subseteq \operatorname{int} C$ converging to 0 such that

$$
\begin{gathered}
f\left(x_{\alpha}, y, \lambda_{\alpha}\right)+e_{\alpha} \nless 0, \text { for all } y \in K_{2}\left(x_{\alpha}, \lambda_{\alpha}\right), \\
\left(f\left(x_{\alpha}, y, \lambda_{\alpha}\right)+e_{\alpha} \geq 0, \text { for all } y \in K_{2}\left(x_{\alpha}, \lambda_{\alpha}\right), \text { respectively }\right) .
\end{gathered}
$$

Definition 3.2. The problem (WQEP) ((SQEP), respectively) is said to be wellposed under perturbations if:

(a) the solution set $S^{w}$ of (WQEP) ( $S^{s}$ of (SQEP), respectively) is nonempty; 
(b) for any net $\left\{\lambda_{\alpha}\right\} \subseteq \Lambda$ converging to $\bar{\lambda}$, every asymptotically solving net for (WQEP) ((SQEP), respectively) corresponding to $\left\{\lambda_{\alpha}\right\}$ has a subnet converging to some point of $S^{w}$ ( $S^{s}$, respectively).

We consider also the following stronger property with the uniqueness requirement as in classical definitions.

Definition 3.3. The problem (WQEP) ((SQEP), respectively) is said to be uniquely well-posed under perturbations if:

(a) there exists a unique solution $\bar{x}$ to (WQEP) ((SQEP), respectively);

(b) for any net $\left\{\lambda_{\alpha}\right\} \subseteq \Lambda$ converging to $\bar{\lambda}$, every asymptotically solving net for (WQEP) ((SQEP), respectively) corresponding to $\left\{\lambda_{\alpha}\right\}$ converges to $\bar{x}$.

From now on, for $\lambda \in \Lambda$ and $e \in \operatorname{int} C$, let $E(\lambda)$ be the set of all fixed points of $K_{1}(., \lambda)$, i.e., the set $\left\{x \in A: x \in K_{1}(x, \lambda)\right\}$, and

$$
\begin{gathered}
\Pi^{w}(\lambda, e)=\left\{x \in K_{1}(x, \lambda): f(x, y, \lambda)+e \nless 0, \forall y \in K_{2}(x, \lambda)\right\}, \\
\Pi^{s}(\lambda, \varepsilon)=\left\{x \in K_{1}(x, \lambda): f(x, y, \lambda)+e \geq 0, \forall y \in K_{2}(x, \lambda)\right\}
\end{gathered}
$$

$\left(\Pi^{w}(\lambda, e)\right.$ and $\Pi^{s}(\lambda, e)$ are $e$-approximate solution sets of $\left(\operatorname{WQEP}_{\lambda}\right)$ and $\left(\left(\operatorname{SQEP}_{\lambda}\right)\right.$, respectively).

\section{Remark 3.1.}

(i) If (WQEP) (or (SQEP)) is (uniquely) well-posed under perturbations, it is Tikhonov well-posed (simply take $\lambda_{\alpha}=\bar{\lambda}$ for all $\alpha$ ).

(ii) The following facts are well-known and often used in sensitivity analysis (see e.g., [1-4]): Let $Q: X \rightarrow 2^{Y}$ be a multimap between two topological spaces.

(ii $i_{a}$ ) If $Q(\bar{x})$ is compact, then $Q($.$) is usc at \bar{x}$ if and only if for any nets $\left\{\bar{x}_{\alpha}\right\} \subseteq X$ with $x_{\alpha} \rightarrow \bar{x}$ and $\left\{y_{\alpha}\right\}$ with $y_{\alpha} \in Q\left(x_{\alpha}\right)$, there is a subnet $\left\{y_{\beta}\right\}$ of $\left\{y_{\alpha}\right\}$ such that $y_{\beta} \rightarrow y$ for some $y \in Q(\bar{x})$.

(ii $b$ ) If, in addition, $Q(\bar{x})=\{\bar{y}\}$ is a singleton then, for the above nets, one has $y_{\alpha} \rightarrow \bar{y}$.

Thus, the definition of well-posedness under perturbations and unique wellposedness under perturbations encompass a form of upper semicontinuous dependence of solutions on the parameter. Such a dependence is investigated in stability (for recent results for quasiequilibrium problems see e.g., [1-4]).

(iii) In [22] the special case, where $K_{1}(x, \lambda) \equiv K_{2}(x, \lambda) \equiv A$ is considered. The definition of parametric well-posedness in [22] is different from Definitions 3.2 and 3.3, since the statements are for all $\lambda \in \Lambda$ instead of for $\bar{\lambda}$ corresponding to the unperturbed problem. Our definitions are similar to those in $[24$, 
$27,38,41]$ and describe more reasonably perturbations of an original problem. However, our results below can be easily restated for the well-posedness defined in [22].

(iv) Well-posedness for vector problems have been defined in various ways. In [9] for instance, for asymptotically solving nets, $e_{\alpha}$ is restricted to being $\epsilon_{\alpha} e$ for $\epsilon_{\alpha}$ being positive numbers and $e \in \operatorname{int} C$, i.e., only fixed direction $e$ is allowed. [17] uses also the scalarizing function, for $y \in Y$,

$$
\xi(y)=\inf \{t: y \leq t e\}
$$

to define well-posedness (called well-posedness of type III there).

A sufficient condition for the well-posedness under perturbations of problem (WQEP) is given by the following theorem. We see that the proof is based on techniques of stability considerations (cf. e.g., [1-4]), since well-posedness under perturbations has close links to stability.

Theorem 3.1. Assume for (WQEP) that $S^{w}$ is nonempty and

(i) $f$ is $C$-usc in $A \times A \times\{\bar{\lambda}\}$;

(ii) either of the following two conditions holds

(ii $\left.1_{1}\right) K_{1}$ is usc in $A \times\{\bar{\lambda}\}, A$ is compact and $K_{2}$ is lsc in $A \times\{\bar{\lambda}\}$;

(iii $) E$ is usc at $\bar{\lambda}$ and $E(\bar{\lambda})$ is compact, and $K_{2}$ is lsc in $A \times\{\bar{\lambda}\}$.

Then, (WQEP) is well-posed under perturbations. Furthermore, if $S^{w}$ is a singleton, then this problem is uniquely well-posed under perturbations.

Proof. It suffices to consider the case ( $\left.\mathrm{ii}_{2}\right)$, since the other case is similar and simpler. We show first that $\Pi^{w}$ is usc at $(\bar{\lambda}, 0)$. Suppose the existence of an open superset $U$ of $\Pi^{w}(\bar{\lambda}, 0)$, of nets $\left\{\left(\lambda_{\alpha}, e_{\alpha}\right)\right\}$ in $\Lambda \times \operatorname{int} C$ with $\left(\lambda_{\alpha}, e_{\alpha}\right) \rightarrow(\bar{\lambda}, 0)$ and of $\left\{x_{\alpha}\right\}$ in $\Pi^{w}\left(\lambda_{\alpha}, e_{\alpha}\right)$ such that $x_{\alpha} \notin U$, for all $\alpha$. By the upper semicontinuity of $E$ at $\bar{\lambda}$ and compactness of $E(\bar{\lambda})$ one can assume that $x_{\alpha} \rightarrow x_{0}$ for some $x_{0} \in E(\bar{\lambda})$. If $x_{0} \notin \Pi^{w}(\bar{\lambda}, 0)=S^{w}(\bar{\lambda})=S^{w}$, then there is $y_{0} \in K_{2}\left(x_{0}, \bar{\lambda}\right)$ such that $f\left(x_{0}, y_{0}, \bar{\lambda}\right)<0$. The lower semicontinuity of $K_{2}$ in turn shows the existence of $y_{\alpha} \in K_{2}\left(x_{\alpha}, \lambda_{\alpha}\right)$ such that $y_{\alpha} \rightarrow y_{0}$. Since $x_{\alpha} \in \Pi^{w}\left(\lambda_{\alpha}, e_{\alpha}\right)$, one has

$$
f\left(x_{\alpha}, y_{\alpha}, \lambda_{\alpha}\right)+e_{\alpha} \nless 0 .
$$

Let $I: C \rightarrow C$ be the identity map. Then Proposition 2.4 yields the $C$-upper semicontinuity of $(x, y, \lambda, e) \rightarrow f(x, y, \lambda)+e$ at $\left(x_{0}, y_{0}, \bar{\lambda}, 0\right)$. Hence

$$
f\left(x_{0}, y_{0}, \bar{\lambda}\right) \nless 0 \text {. }
$$


which is a contradiction. Hence, $x_{0} \in \Pi^{w}(\bar{\lambda}, 0) \subseteq U$, which is again a contradiction, since $x_{\alpha} \notin U$, for all $\alpha$. Thus, $\Pi^{w}$ is usc at $(\bar{\lambda}, 0)$.

Next we check that $S^{w}$ is compact by showing simply its closedness (as $E(\bar{\lambda})$ is compact). Let $x_{\alpha} \in S^{w}$ with $x_{\alpha} \rightarrow x_{0}$. If $x_{0} \notin S^{w}$, there exists $y_{0} \in K_{2}\left(x_{0}, \bar{\lambda}\right)$ such that

$$
f\left(x_{0}, y_{0}, \bar{\lambda}\right)<0 .
$$

The lower semicontinuity of $K_{2}$ gives the existence of $y_{\alpha} \in K_{2}\left(x_{\alpha}, \bar{\lambda}\right)$ such that $y_{\alpha} \rightarrow y_{0}$. Since $x_{\alpha} \in S^{w}$ for each $\alpha$, one has

$$
f\left(x_{\alpha}, y_{\alpha}, \bar{\lambda}\right) \nless 0 .
$$

The $C$-upper semicontinuity of $f(., ., \bar{\lambda})$ now implies that

$$
f\left(x_{0}, y_{0}, \bar{\lambda}\right) \nless 0,
$$

which is impossible. Consequently, $S^{w}$ is compact.

Finally, taking Remark $3.1\left(\mathrm{ii}_{a}\right.$ ) into account, we see that the upper semicontinuity of $\Pi^{w}$ and the compactness of $S^{w}$ together imply the well-posedness under perturbations of (WQEP). In the particular case, where $S^{w}$ is a singleton, the unique well-posedness under perturbations is followed by Remark 3.1 (ii $b$ ).

Remark 3.2. In the special case, where $K_{1}(x, \lambda) \equiv K_{2}(x, \lambda) \equiv A$, assumption (i) of Theorem 3.1 can clearly be reduced to the $C$-upper semicontinuity of $f(., y,$.$) ,$ for each $y \in A$. Therefore, this theorem contains the well-posedness part of Theorem 3.3 and Corollary 3.1 of [22] (with abuse of the fact that the latter paper considers well-posedness for all $\lambda \in \Lambda$, since such results can be obtained by modifying Theorem 3.1). The other part of that theorem is about the existence of solutions for all $\lambda \in \Lambda$. But in our approach both in the definition of well-posedness and in Theorem 3.1, we need the existence of solutions only for $\bar{\lambda}$. Moreover, in the literature there have been many papers on the solution existence for quasiequilibrium problems (unparametrized, or what is the same, depending on one fixed parameter $\bar{\lambda})$. Therefore, we focus on well-posedness, leaving our needed existence as an assumption.

The following example shows that, even in the mentioned special case, the $C$ upper semicontinuity in assumption (i) should be imposed with respect to (shortly, wrt) both $x$ and $\lambda$.

Example 3.1. Let $X=Y=R, \Lambda=[0,1], \bar{\lambda}=0, A=[0,1], C=R_{+}, K_{1}(x, \lambda)$ $=K_{2}(x, \lambda)=[0,1]$ and

$$
f(x, y, \lambda)= \begin{cases}x-y & \text { if } \lambda=0 \\ y-x & \text { otherwise }\end{cases}
$$


Then assumption ( $\mathrm{ii}_{1}$ ) is satisfied. As for (i), $f$ is continuous wrt $x$ but not $R_{+}$-usc wrt $\lambda$. Indeed, taking $x_{n}=0, y_{n}=1, \lambda_{n}=\frac{1}{n}$ and $e_{n}=0$, we have $\left(x_{n}, y_{n}, \lambda_{n}, e_{n}\right) \rightarrow(0,1,0,0)$ and $f\left(x_{n}, y_{n}, \lambda_{n}\right)+e_{n}=f\left(0,1, \frac{1}{n}\right)=1>0$, but $f(0,1,0)=-1<0$. We see directly that $S(0)=\{1\}$ and, for $\lambda_{n}=\frac{1}{n}, e_{n}=\frac{1}{n}$, the sequence $\left\{x_{n}\right\}$ in $\Pi\left(\lambda_{n}, e_{n}\right)$, with $x_{n}=0$ for all $n$, is an asymptotically solving sequence for (QEP) corresponding to $\lambda_{n}$ and $e_{n}$. But $x_{n} \rightarrow 0 \notin S(0)$ and hence (QEP) is not well-posed under perturbations.

For the strong problem (SQEP) we have the following result.

Theorem 3.2. Impose the assumptions of Theorem 3.1 except (i), which is replaced by

(i') $f$ is $C$-qusc in $A \times A \times\{\bar{\lambda}\}$.

Then, (SQEP) is well-posed under perturbations. Furthermore, if $S^{s}$ is a singleton, then (SQEP) is uniquely well-posed under perturbations.

Proof. The proof is technically similar to that of Theorem 3.1. We present it shortly for the sake of completeness. To prove first the upper semicontinuity of $\Pi^{s}$ by contraposition, suppose the existence of an open superset $U$ of $\Pi^{s}(\bar{\lambda}, 0)$ and nets $\left\{\left(\lambda_{\alpha}, e_{\alpha}\right)\right\}$ and $\left\{x_{\alpha}\right\}$ in $\Pi^{s}\left(\lambda_{\alpha}, e_{\alpha}\right)$ such that $\left(\lambda_{\alpha}, e_{\alpha}\right) \rightarrow\left(\lambda_{0}, 0\right)$, and $x_{\alpha} \notin U$, for all $\alpha$. By assumption (ii) one can assume that $x_{\alpha} \rightarrow x_{0}$ for some $x_{0} \in \Pi^{s}(\bar{\lambda}, 0)$, a contradiction. (Instead of Proposition 2.4, here we use the sum rule in Proposition 2.5 (ii) for $C$-qusc function $f$.)

Next suppose for $x_{\alpha} \in S^{s}$, one has $x_{\alpha} \rightarrow x_{0}$ but $x_{0} \notin S^{s}$. Then, there exists $y_{0} \in K_{2}\left(x_{0}, \bar{\lambda}\right)$ such that

$$
f\left(x_{0}, y_{0}, \bar{\lambda}\right) \geq 0 .
$$

The lower semicontinuity of $K_{2}$ and the $C$ - quasi upper semicontinuity of $f(., ., \bar{\lambda})$ together imply the contradiction that

$$
f\left(x_{0}, y_{0}, \bar{\lambda}\right) \geq 0 .
$$

Therefore, $S^{s}$ is closed and hence compact.

Finally, by Remark 3.1 (ii), the upper semicontinuity of $\Pi^{s}$ and compactness or being a singleton of $S^{s}$ together complete the proof.

\section{QuAsiconveXity of VeCtor Functions}

Now we discuss several generalized quasiconvexity notions for vector functions and develop their properties in order to use them for our well-posedness study in the next section. In this section let $X$ be a vector space and $Y$ be a topological vector space partially ordered by a solid pointed closed convex cone $C, A \subseteq X$ be a convex set and $h: A \rightarrow Y$. The following definition is known. (The relations $\leq$, $<$, etc, are defined by $C$.) 
Definition 4.1. (i) $h$ is called convex if, for every $x_{1}, x_{2} \in A$ and $t \in[0,1]$,

$$
h\left(t x_{1}+(1-t) x_{2}\right) \leq t h\left(x_{1}\right)+(1-t) h\left(x_{2}\right) .
$$

(ii) $h$ is said to be strictly convex if, for every $x_{1}, x_{2} \in A$ and $t \in(0,1)$,

$$
h\left(t x_{1}+(1-t) x_{2}\right)<t h\left(x_{1}\right)+(1-t) h\left(x_{2}\right) .
$$

(iii) $h$ is said to be quasiconvex if, for every $x_{1}, x_{2} \in A$ and $t \in[0,1]$, either

$$
h\left(t x_{1}+(1-t) x_{2}\right) \leq h\left(x_{1}\right) \quad \text { or } \quad h\left(t x_{1}+(1-t) x_{2}\right) \leq h\left(x_{2}\right) .
$$

(iv) $h$ is said to be strictly quasiconvex if, for every $x_{1}, x_{2} \in A$ and $t \in(0,1)$, either

$$
h\left(t x_{1}+(1-t) x_{2}\right)<h\left(x_{1}\right) \quad \text { or } \quad h\left(t x_{1}+(1-t) x_{2}\right)<h\left(x_{2}\right) .
$$

For these definitions and for all later convexity-related definitions, by inverting the inequalities we get the corresponding concavity notions. In other words, $h$ is concave (strictly concave, quasiconcave, etc) if and only if $-h$ is convex (strictly convex, quasiconvex, etc, respectively).

Remark 4.1. If $Y=C \cup-C$, like for scalar functions, convexity (strict convexity, respectively) implies quasiconvexity (strict quasiconvexity). However, this is not true in general as shown by the following example.

Example 4.1. Let $X=A=R, Y=R^{2}, C=R_{+}^{2}$ and $h(x)=(-1-$ $2 x,-3+3 x)$. Then $h$ is convex as it is linear. But $h$ is not quasiconvex. Indeed, for $x_{1}=0, x_{2}=1, t=\frac{1}{2}$, one has $h\left(x_{1}\right)=(-1,-3), h\left(x_{2}\right)=(-3,0)$, but $h\left(\frac{1}{2} x_{1}+\frac{1}{2} x_{2}\right)=\left(-2,-\frac{3}{2}\right) \not \leq h\left(x_{1}\right)$ and $\not \leq h\left(x_{2}\right)$. In [14, pp. 48-49] there is the assertion that convexity implies quasiconvexity for general set-vector-valued functions. This example shows that this assertion is not adequate even for the single-valued case.

The following example shows that, for vector functions, even strict convexity does not imply quasiconvexity.

Example 4.2. Let $X=A=R, Y=R^{2}, C=R_{+}^{2}$ and $h(x)=\left(4 x^{2}+\right.$ $\left.2 x-1,4 x^{2}-10 x+5\right):=\left(h_{1}(x), h_{2}(x)\right)$. Observe that both $h_{1}$ and $h_{2}$ are strictly convex scalar functions. Hence, for all $x_{1}, x_{2} \in R$, all $t \in(0,1)$ and for $x_{t}=t x_{1}+(1-t) x_{2}$, one has

$$
h\left(x_{t}\right)<t h\left(x_{1}\right)+(1-t) h\left(x_{2}\right),
$$

i.e., $h$ is strictly convex. But $h$ is not quasiconvex. Indeed, for $x_{1}=0, x_{2}=1, t=$ $\frac{1}{2}$, one has $h\left(x_{1}\right)=(-1,5), h\left(x_{2}\right)=(5,-1)$ and $h\left(\frac{1}{2} x_{1}+\frac{1}{2} x_{2}\right)=(1,1) \not \leq h\left(x_{1}\right)$ and $\not \leq h\left(x_{2}\right)$. 
Definition 4.2. Let $b \in Y$ be fixed. If, for all $x_{1}, x_{2} \in A$, all $t \in(0,1)$ and for $x_{t}=(1-t) x_{1}+t x_{2}$,

(i) $h\left(x_{1}\right) \nless b, h\left(x_{2}\right) \not \leq b$ implies $h\left(x_{t}\right) \not \leq b$, then $h$ is called lev $\not b$-convex;

(ii) $h\left(x_{1}\right) \geq b, h\left(x_{2}\right)>b$ implies $h\left(x_{t}\right)>b$, then $h$ is said to be lev $>b$-convex.

Note that, if $Y=C \cup-C$, these two properties coincide. Furthermore, if $h$ is $\operatorname{lev}_{\not b}$-convex (lev $>b$-convex, respectively), then $L_{\not \leq b} h$ ( $L_{>b} h$, respectively) is convex, but not vice versa as shown by the following example.

Example 4.3. Let $A=[-2,2], Y=R, C=R_{+}$and $h(x)=\max \{0, x\}$ for $x \in A$. Then $\mathrm{L}_{\measuredangle 0} h=(0,2]$ is convex, but $h$ is not $\operatorname{lev}_{\measuredangle 0}$-convex. Indeed, let $x_{1}=-1, x_{2}=1$ and $t=\frac{1}{2}$. Then $h\left(x_{1}\right)=0 \nless 0, h\left(x_{2}\right)=1 \not \leq 0$, but $h\left(x_{t}\right)=h\left(\frac{1}{2} x_{1}+\frac{1}{2} x_{2}\right)=h(0)=0 \leq 0$.

Remark 4.2. If $h$ is strictly quasiconcave, then $h$ is $\operatorname{lev}_{\not b}$-convex, for all $b \in Y$. Indeed, let $x_{1}, x_{2} \in A$ be such that $h\left(x_{1}\right) \nless b, h\left(x_{2}\right) \not \leq b$. Suppose the existence of $c_{1} \in C$ such that $h\left(x_{t}\right)-b=-c_{1}$. Since $h$ is strictly quasiconcave, $h\left(x_{t}\right)>h\left(x_{1}\right)$ or $h\left(x_{t}\right)>h\left(x_{2}\right)$, for all $t \in(0,1)$. If $h\left(x_{t}\right)>h\left(x_{1}\right)$, there is $c_{2} \in \operatorname{int} C$ with $h\left(x_{t}\right)=h\left(x_{1}\right)+c_{2}$. Hence, $h\left(x_{1}\right)-b=-c_{1}-c_{2} \in-\operatorname{int} C$, which is impossible as $h\left(x_{1}\right) \nless b$. If $h\left(x_{t}\right)>h\left(x_{2}\right)$ we have a similar contradiction.

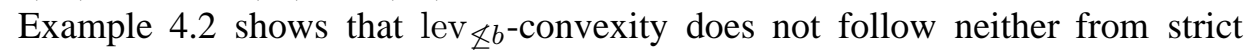
concavity nor from strict convexity. Indeed, we know that $h(x)=\left(4 x^{2}+2 x-\right.$ $\left.1,4 x^{2}-10 x+5\right)$ is strictly convex (and $-h$ is strictly concave). For $b=0, x_{1}=$ $0, x_{2}=1$ and $t=\frac{1}{2}$, we have $h\left(x_{1}\right)=(-1,5) \nless 0, h\left(x_{2}\right)=(5,-1) \not \leq 0$, but $h\left(x_{\frac{1}{2}}\right)=(1,1) \leq 0$. Similarly, $-h$ is not $\operatorname{lev}_{\not b}$-convex either. The next example indicates that neither quasiconcavity nor quasiconvexity implies lev $\underset{\not}{ } b^{-c o n v e x i t y . ~}$

Example 4.4. Let $X=Y=R, A=R, C=R_{+}$and

$$
h(x)= \begin{cases}0 & \text { if } x \leq 1 \\ x-1 & \text { otherwise }\end{cases}
$$

Then, $h$ is both quasiconvex and quasiconcave. But $h$ is not lev $\measuredangle 0$-convex, since for $x_{1}=0, x_{2}=2$, one has $h\left(x_{1}\right)=0 \not \leq 1, h\left(x_{2}\right)=1 \nless 1$, but $h\left(x_{\frac{1}{2}}\right)=0 \not \leq 1$.

Remark 4.3. If $h$ is strictly quasiconcave or concave, then $h$ is $\operatorname{lev}_{>b}$-convex for all $b \in Y$. Indeed, assume that $h$ is strictly quasiconcave and $x_{1}, x_{2} \in A$ such that $h\left(x_{1}\right) \geq b, h\left(x_{2}\right)>b$. For any $t \in(0,1)$, if $h\left(x_{t}\right)>h\left(x_{1}\right)$, there is $c_{1} \in \operatorname{int} C$ with $h\left(x_{t}\right)-h\left(x_{1}\right)=c_{1}$. Since $h\left(x_{1}\right) \geq b$, there is $c_{2} \in C$ with $h\left(x_{1}\right)=b+c_{2}$. Consequently, $h\left(x_{t}\right)-b=c_{1}+c_{2} \in \operatorname{int} C$, i.e., $h\left(x_{t}\right)>b$. Similarly, $h\left(x_{t}\right)>b$ if $h\left(x_{t}\right)>h\left(x_{2}\right)$. Thus, $h$ is $\operatorname{lev}_{>b}$-convex. Now assume that $h$ is concave and $x_{1}, x_{2} \in A$ such that $h\left(x_{1}\right) \geq b, h\left(x_{2}\right)>b$. Then, there 
is $c_{1} \in C$ and $c_{2} \in \operatorname{int} C$ such that $h\left(x_{1}\right)=b+c_{1}, h\left(x_{2}\right)=b+c_{2}$. Since $h$ is concave, there exists $c_{3} \in C$ such that $h\left(x_{t}\right)-t h\left(x_{1}\right)-(1-t) h\left(x_{2}\right)=c_{3}$. Hence, $h\left(x_{t}\right)-b=t c_{1}+(1-t) c_{2}+c_{3} \in \operatorname{int} C$. Thus, $h\left(x_{t}\right)>b$.

Example 4.4 shows also that neither quasiconcavity nor quasiconvexity yields $\operatorname{lev}_{>b}$-convexity, since in this case $\operatorname{lev}_{\measuredangle b}$-convexity coincides with $\operatorname{lev}_{>b}$-convexity.

\section{Well-posedness for Equilibrium Problems with Equilibrium Constraints}

Let $X, Y$ and $Z$ be Hausdorff topological vector spaces. $K \subseteq X$ be a nonempty convex subset, $C \subseteq Y$ and $D \subseteq Z$ are solid pointed convex cones, $f: K \times K \rightarrow Y$ and $g: K \times K \rightarrow Z$. Consider the following two equilibrium problems with equilibrium constraints

(WEPEC) Find $\bar{x} \in S_{1}^{w}$ such that, for all $y \in S_{1}^{w}$,

$$
g(\bar{x}, y) \nless 0,
$$

where $S_{1}^{w}=\{x \in K: f(x, y) \nless 0, \forall y \in K\}$;

(SEPEC) Find $\bar{x} \in S_{1}^{s}$ such that, for all $y \in S_{1}^{s}$,

$$
g(\bar{x}, y) \geq 0
$$

where $S_{1}^{s}=\{x \in K: f(x, y, \lambda) \geq 0, \forall y \in K\}$.

These problems can be expressed otherwise as rewritten below (we write for (WEPEC) only)

(WEPEC) Find the value $x=\bar{x}$ such that

$$
\begin{gathered}
g(x, y) \nless 0, \\
f(x, z) \nless 0, f(y, z) \nless 0, \\
z \in K .
\end{gathered}
$$

Assume that the perturbations upon these problems can be expressed in terms of a perturbing parameter $\lambda$ which varies in a Hausdorff topological space $\Lambda$ in the way that we can embed our two problems into the corresponding parametric families as follows. The constraint set $K$ depends on $\lambda$ as a multimap $K: A \times \Lambda \rightarrow 2^{A}$, where $A \subseteq X$ is nonempty. The maps $f$ and $g$ now depend on $\lambda$. The parametric families have the form: 
$\left(\mathrm{WEPEC}_{\lambda}\right.$ ) Find $\bar{x} \in S_{1}^{w}(\lambda)$ such that, for all $y \in S_{1}^{w}(\lambda)$,

$$
g(\bar{x}, y, \lambda) \nless 0,
$$

where $S_{1}^{w}(\lambda)=\{x \in K(\lambda): f(x, y, \lambda) \nless 0, \forall y \in K(\lambda)\}$.

$\left(\operatorname{SEPEC}_{\lambda}\right)$ Find $\bar{x} \in S_{1}^{s}(\lambda)$ such that, for all $y \in S_{1}^{s}(\lambda)$,

$$
g(\bar{x}, y, \lambda) \geq 0,
$$

where $S_{1}^{s}(\lambda)=\{x \in K(\lambda): f(x, y, \lambda) \geq 0, \forall y \in K(\lambda)\}$.

Assume further that the original problems are corresponding to $\bar{\lambda}$. We denote the solutions sets of $\left(\mathrm{WEPEC}_{\lambda}\right)$ and $\left(\mathrm{SEPEC}_{\lambda}\right)$ by $S_{2}^{w}(\lambda)$ and $S_{2}^{s}(\lambda)$, respectively. As auxiliary problems we use also the following "strict" versions of the constraints $S_{1}^{w}$ and $S_{1}^{s}$, respectively, for $\lambda \in \Lambda$,

$$
\begin{gathered}
S_{0}^{w}(\lambda)=\{x \in K(\lambda): f(x, y, \lambda) \not \leq 0, \forall y \in K(\lambda)\}, \\
S_{0}^{s}(\lambda)=\{x \in K(\lambda): f(x, y, \lambda)>0, \forall y \in K(\lambda)\} .
\end{gathered}
$$

We first establish a sufficient condition for the well-posedness of (WEPEC).

Theorem 5.1. Assume for (WEPEC) that $S_{2}^{w}(\bar{\lambda}), S_{0}^{w}(\bar{\lambda})$ are nonempty and

(i) $K$ is continuous at $\bar{\lambda}$ and $K(\bar{\lambda})$ is convex and compact;

(ii) $g$ is $C$-usc in $A \times A \times\{\bar{\lambda}\}$;

(iii) in $A \times A \times\{\bar{\lambda}\}, f$ is 0 -level $C$-qlsc and 0 -level $C$-usc;

(iv) for all $y \in A, L_{\nless 0} f(., y, \bar{\lambda})$ is convex and $f(., y, \bar{\lambda})$ is $\operatorname{lev}_{\not 0}$-convex.

Then, (WEPEC) is well-posed under perturbations. Furthermore, if the solution set $S_{2}^{w}(\bar{\lambda})$ is a singleton, then (WEPEC) is uniquely well-posed under perturbations.

Proof. To apply Theorem 3.1 we need to verify only assumption (ii ${ }_{2}$ ) with $K_{1}()=.K_{2}()=.S_{1}^{w}()=.E($.$) . Let us show first the compactness of S_{1}^{w}$. As $K(\bar{\lambda})$ is compact, we have to show only the closedness. Let $x_{\alpha} \in S_{1}^{w}$ be such that $x_{\alpha} \rightarrow x$. For each $y \in K(\bar{\lambda})$, we have

$$
f\left(x_{\alpha}, y, \bar{\lambda}\right) \nless 0 .
$$

By the closedness of $\mathrm{L}_{\nless 0} f(., y, \bar{\lambda}), f\left(x_{0}, y, \bar{\lambda}\right) \nless 0$, i.e., $x \in S_{1}^{w}$, and hence $S_{1}^{w}$ is closed.

Next we prove that $S_{1}^{w}($.$) is usc at \bar{\lambda}$. Suppose the existence of nets $\left\{\lambda_{\alpha}\right\}$ with $\lambda_{\alpha} \rightarrow \bar{\lambda}$ and $\left\{x_{\alpha}\right\}$ with $x_{\alpha} \in S^{w}\left(\lambda_{\alpha}\right)$, of a superset $U$ of $S^{w}(\bar{\lambda})$ such that $x_{\alpha} \notin U$, 
for all $\alpha$. By the upper semicontinuity of $K($.$) and the compactness of K(\bar{\lambda})$ one can assume that $x_{\alpha} \rightarrow x$, for some $x \in K(\bar{\lambda})$. If $x \notin S_{1}^{w}(\bar{\lambda})$, there is $y \in K(\bar{\lambda})$ such that $f(x, y, \bar{\lambda})<0$. Since $K$ is lsc at $\bar{\lambda}$, there exists $y_{\alpha} \in K\left(\lambda_{\alpha}\right)$ such that $y_{\alpha} \rightarrow y$. Since $x_{\alpha} \in S_{1}^{w}\left(\lambda_{\alpha}\right)$,

$$
f\left(x_{\alpha}, y_{\alpha}, \lambda_{\alpha}\right) \nless 0 .
$$

As $f$ is 0 -level $C$-usc, $f(x, y, \bar{\lambda}) \nless 0$, which is impossible. Thus, $x \in S_{1}^{w}(\bar{\lambda}) \subseteq U$, which is again a contradiction, since $x_{\alpha} \notin U$, for all $\alpha$.

To verify now the lower semicontinuity of $S_{1}^{w}($.$) we prove first that S_{0}^{w}($.$) is$ lsc at $\bar{\lambda}$. Suppose to the contrary that there exist $x_{0} \in S_{0}^{w}(\bar{\lambda})$ and $\lambda_{\alpha} \rightarrow \bar{\lambda}$ such that, for all $x_{\alpha} \in S_{0}^{w}\left(\lambda_{\alpha}\right), x_{\alpha} \nrightarrow x_{0}$. Since $K$ is lsc at $\bar{\lambda}$, there exists $x_{\alpha} \in K\left(\lambda_{\alpha}\right)$ such that $x_{\alpha} \rightarrow x_{0}$. By the above contradiction assumption, we can assume that $x_{\alpha} \notin S_{0}^{w}\left(\lambda_{\alpha}\right)$ for all $\alpha$, i.e., for some $y_{\alpha} \in K\left(\lambda_{\alpha}\right)$,

$$
f\left(x_{\alpha}, y_{\alpha}, \lambda_{\alpha}\right) \leq 0 .
$$

By assumption (i) we can assume that $y_{\alpha} \rightarrow y_{0}$ for some $y_{0} \in K(\bar{\lambda})$. The closedness of $\mathrm{L}_{\leq 0} f$ and (1) together yield that $f\left(x_{0}, y_{0}, \bar{\lambda}\right) \leq 0$, which is impossible since $x_{0} \in S_{0}^{w}(\bar{\lambda})$.

Now let us check that

$$
S_{1}^{w}(\bar{\lambda}) \subseteq \operatorname{cl} S_{0}^{w}(\bar{\lambda})
$$

Let $x_{1} \in S_{1}^{w}(\bar{\lambda}), x_{0} \in S_{0}^{w}(\bar{\lambda}), t \in(0,1)$ and $x_{t}=(1-t) x_{1}+t x_{0}$. Since $K(\bar{\lambda})$ is convex, $x_{t} \in K(\bar{\lambda})$. We show that $x_{t} \in S_{0}^{w}(\bar{\lambda})$. For each $y \in K(\bar{\lambda})$, we have $f\left(x_{0}, y, \bar{\lambda}\right) \not \leq 0$ and $f\left(x_{1}, y, \bar{\lambda}\right) \nless 0$. Since $f(., y, \bar{\lambda})$ is $\operatorname{lev}_{\not \geq 0}$-convex,

$$
f\left(x_{t}, y, \bar{\lambda}\right) \not \leq 0,
$$

i.e., $x_{t} \in S_{0}^{w}(\bar{\lambda})$. Therefore (2) holds. By the lower semicontinuity of $S_{0}^{w}$ at $\bar{\lambda}$, if $\lambda_{\alpha} \rightarrow \bar{\lambda}$ we have

$$
S_{1}^{w}(\bar{\lambda}) \subseteq \operatorname{cl} S_{0}^{w}(\bar{\lambda}) \subseteq \liminf S_{0}^{w}\left(\lambda_{\alpha}\right) \subseteq \liminf S_{1}^{w}\left(\lambda_{\alpha}\right),
$$

i.e., $S_{1}^{w}$ is lsc at $\bar{\lambda}$.

Finally, applying Theorem 3.1 completes the proof.

Since the assumptions of Theorem 5.1 look complicated we provide now examples showing their essentialness.

Example 5.1. ((i) is essential). Let $X=Y=R, A=[-1,1], \Lambda=[0,1], C=$ $R_{+}, \bar{\lambda}=0$,

$$
K(\lambda)= \begin{cases}{[-1,1]} & \text { if } \lambda=0 \\ {[0,1]} & \text { otherwise }\end{cases}
$$


and $f(x, y, \lambda)=x+y+2, g(x, y, \lambda)=x+y+\lambda$. It is clear that $K(\lambda)$ is usc at $0, K(0)$ is compact, assumptions (ii)-(iv) hold since $f$ and $g$ are continuous and $f(., y, \lambda)$ is convex. We have $S_{1}^{w}(0)=[-1,1], S_{2}^{w}(0)=\{1\}$ and, for all $\lambda \in(0,1], S_{1}^{w}(\lambda)=[0,1]=S_{2}^{w}(\lambda)\left(S_{2}^{w}(\lambda)\right.$ is the solution set of $\left.\left(\mathrm{WEPEC}_{\lambda}\right)\right)$. Taking $\lambda_{n}=e_{n}=\frac{1}{n}$ we see that $x_{n}=0$ is an asymptotically solving sequence for (WEPEP) corresponding to $\lambda_{n}$ and $e_{n}$. But $x_{n} \rightarrow 0 \notin S_{2}^{w}(0)$ and hence (WEPEC) is not well-posed under perturbations. The reason is that (i) is not satisfied, since $K$ is not lsc at 0 .

Example 5.2. ((ii) cannot be dropped) Let $X=Y=R, A=[-1,1], \Lambda=$ $[0,1], C=R_{+}, \bar{\lambda}=0, K(\lambda)=[-1,1], f(x, y, \lambda)=x$ and

$$
g(x, y, \lambda)= \begin{cases}x-y & \text { if } \lambda=0 \\ y-x & \text { otherwise }\end{cases}
$$

Assumptions (i), (iii) and (iv) evidently hold. We see that $S_{2}^{w}(0)=\{1\}$ and $S_{2}^{w}(\lambda)=\{0\}$ for all $\lambda \in(0,1] . x_{n}=0$ is clearly an asymptotically solving sequence for (WEPEP) corresponding to $\lambda_{n}=e_{n}=\frac{1}{n}$. Then, (WEPEC) is not well-posed under perturbations as $x_{n} \rightarrow 0 \notin S_{2}^{w}(0)$. The reason is that (ii) is violated. Indeed, let $x_{n}=0, y_{n}=1$ and $\lambda_{n}=\frac{1}{n}$. Then, $\left(x_{n}, y_{n}, \lambda_{n}\right) \rightarrow(0,1,0)$ and $g\left(x_{n}, y_{n}, \lambda_{n}\right)=1 \geq 0$, but $g(0,1,0)=-1<0$.

Example 5.3. ((iii) cannot be dispensed) Let $X=Y=R, A=[-1,1], \Lambda=$ $[0,1], C=R_{+}, \bar{\lambda}=0, K(\lambda)=[-1,1], g(x, y, \lambda)=1$ and

$$
f(x, y, \lambda)= \begin{cases}-1 & \text { if } x \leq 0 \\ 1 & \text { otherwise }\end{cases}
$$

It is obvious that assumptions (i), (ii) and (iv) hold. We see that $S_{2}^{w}(\lambda)=(0,1]$ for all $\lambda \in[0,1]$, and $x_{n}=\frac{1}{n}$ is an asymptotically solving sequence for (WEPEP) corresponding to $\lambda_{n}=e_{n}=\frac{1}{n}$. But $x_{n} \rightarrow 0 \notin S_{2}^{w}(0)$ and hence (WEPEC) is not well-posed under perturbations. This is not surprising since (iii) is not fulfilled. Indeed, let $x_{n}=\frac{1}{n}, y_{n}=1$ and $\lambda_{n}=\frac{1}{n}$. Then, $\left(x_{n}, y_{n}, \lambda_{n}\right) \rightarrow(0,1,0)$ and $f\left(x_{n}, y_{n}, \lambda_{n}\right)=1 \geq 0$, but $f(0,1,0)=-1<0$, and hence $f$ is not $(0, C)$-usc at $(0,1,0)$.

Example 5.4. ((iv) is essential) Let $X=Y=R, A=[-1,3], \Lambda=[0,1], C=$ $R_{+}, \bar{\lambda}=0, K(\lambda)=[-1,3], f(x, y, \lambda)=-x\left(x^{2}-1-\lambda\right)$ and $g(x, y, \lambda)=$ $x+y+\lambda$. Assumptions (i)-(iv) are apparently fulfilled. We easily compute that $S_{1}^{w}(0)=\{-1\} \cup[0,1], S_{2}^{w}(0)=\{1\}$ and, for all $\lambda \in(0,1], S_{1}^{w}(\lambda)=[0, \sqrt{1+\lambda}]$ and $S_{2}^{w}(\lambda)=[0, \sqrt{1+\lambda}]$. Taking $\lambda_{n}=e_{n}=\frac{1}{n}$ one sees that $x_{n}=0$ is an asymptotically solving sequence for (WEPEP) corresponding to $\lambda_{n}=e_{n}=\frac{1}{n}$. But 
$x_{n} \rightarrow 0 \notin S_{2}^{w}(0)$, whence (WEPEC) is not well-posed under perturbations. The reason is that (iv) is violated. Indeed, taking $x_{1}=-1, x_{2}=\frac{1}{2}, t=\frac{2}{3}$ and $y=0$, we see that $f\left(x_{1}, y, 0\right)=0, f\left(x_{2}, y, 0\right)=\frac{3}{8}$, but $f\left(x_{t}, y, 0\right)=f\left(-\frac{1}{2}, y, 0\right)=-\frac{3}{8}<0$.

Theorem 5.2. Assume for (SEPEC) that $S_{2}^{s}(\bar{\lambda}), S_{0}^{s}(\bar{\lambda})$ are nonempty and assume further that

(i) $K$ is continuous at $\bar{\lambda}$ and $K(\bar{\lambda})$ is convex and compact;

(ii) $g$ is $C$-qusc in $A \times A \times\{\bar{\lambda}\}$;

(iii) in $A \times A \times\{\bar{\lambda}\}, f$ is 0 -level $C$-lsc and 0-level $C$-qusc;

(iv) for all $y \in A, f(., y, \bar{\lambda})$ is $\operatorname{lev}_{>0}$-convex.

Then, (SEPEC) is well-posed under perturbations. Furthermore, if the solution set $S_{2}^{s}(\bar{\lambda})$ is a singleton, then (SEPEC) is uniquely well-posed under perturbations.

Proof. We have to verify only assumption (iii $)$ of Theorem 3.2 in order to apply it. First, the compactness of $S_{1}^{s}$ is checked similarly as that of $S_{1}^{w}$, but using the 0-level $C$-quasi upper semicontinuity of $f$. Next, the upper semicontinuity of $S_{1}^{s}$ is verified analogously as the counterpart of $S_{1}^{w}$. For the lower semicontinuity of $S_{1}^{s}$, we show first this property for the map $S_{0}^{s}$. Then, via proving the inclusion

$$
S_{1}^{s}(\lambda) \subseteq \operatorname{cl} S_{0}^{s}(\lambda)
$$

by using the assumed $\operatorname{lev}_{>0}$-convexity of $f(., y, \bar{\lambda})$ we get the required semicontinuity.

\section{ACKNOWLEDGMENT}

This work was completed during a scientific stay of the second author at the Department of Applied Mathematics, National Sun Yat-Sen University, Kaohsiung, Taiwan, Republic of China. The hospitality and financial support are gratefully acknowledged. The fourth author was partially supported by the grant NSC 972115-M-110-001.

\section{REFERENCES}

1. L. Q. Anh and P. Q. Khanh, Semicontinuity of the solution sets of parametric multivalued vector quasiequilibrium problems, J. Math. Anal. Appl., 294 (2004), 699-711.

2. L. Q. Anh and P. Q. Khanh, On the stability of the solution sets of general multivalued vector quasiequilibrium problems, J. Optim. Theory Appl., 135 (2007), 271-284. 
3. L. Q. Anh and P. Q. Khanh, Various kinds of semicontinuity and the solutions ets of parametric multivalued symmetric vector quasiequilibrium problems, J. Glob. Optim., 41 (2008), 539-558.

4. L. Q. Anh and P. Q. Khanh, Semicontinuity of the approximate solution sets of multivalued quasiequilibrium problems, Numer. Funct. Anal. Optim., 29 (2008), 24-42.

5. H. Attouch and R. Wets, Quantitative stability of variational systems, Trans. Amer. Math. Soc., 328 (1991), 695-730.

6. G. Beer and R. Lucchetti, The epi-distance topology, Math. Oper. Res., 17 (1992), 715-726.

7. L. C. Ceng, N. Hadjisavvas, S. Schaible and J. C. Yao, Well-posedness for mixed quasivariational-like inequalities, J. Optim. Theory Appl., 139 (2008), 109-125.

8. L. C. Ceng and J. C. Yao, Well-posedness of generalized mixed variational inequalities, inclusion problems and fixed point problems, Nonlinear Anal. TMA, 69 (2008), 4585-4603.

9. G. P. Crespi, A. Guerraggio and M. Rocca, Well-posedness in vector optimization problems and vector variational inequalities, J. Optim. Theory Appl., 132 (2007), 213-226.

10. J. Demmel, On condition numbers and the distance of the nearest ill-posed problem, Numer. Math., 51 (1987), 251-289.

11. Y. P. Fang, N. J. Huang and J. C. Yao, Well-posedness of mixed variational inequalities, inclusion problems and fixed point problems, J. Glob. Optim., 41 (2008), 117-133.

12. Y. P. Fang, R. Hu and N.J. Huang, Well-posedness for equilibrium problems and for optimization problems with equilibrium constraints, Computers Math. Appl., 55 (2008), 89-100.

13. M. Furi and A. Vignoli, About well-posed optimization problem for functionals in metric spaces, J. Optim. Theory Appl., 5 (1970), 225-229.

14. A. Göpfert, C. Tammer, H. Riahi and C. Zalinescu, Variational Methods in Partially Ordered Spaces, Springer, Berlin, Germany, 2003.

15. J. Hadamard, Sur le problèmes aux dérivees partielles et leur signification physique, Bull. Univ. Princeton, 13 (1902), 49-52.

16. X. X. Huang and X. Q. Yang, Gereralized Levitin-Polyak well-posedness in constrained optimization, SIAM J. Optim., 17 (2006), 243-258.

17. X. X. Huang and X. Q. Yang, Levitin-Polyak well-posedness of constrained vector optimization problems, J. Glob. Optim., 37 (2007), 287-304.

18. A. Ioffe and R. E. Lucchetti, Typical convex program is very well-posed, Math. Prog., Ser. B, 104 (2005), 483-499. 
19. A. Ioffe, R. E. Lucchetti and J. P. Revalski, A variational principle for problems with functional constraints, SIAM J. Optim., 12 (2001), 461-478.

20. A. Ioffe, R. E. Lucchetti and J. P. Revalski, Almost every convex or quadratic programming problem is well-posed, Math. Oper. Res., 29 (2004), 369-382.

21. P. S. Kenderov and J. P. Revalski, Generic well-posedness of optimization problems and Banach-Mazur game, in: Recent Developments in Well-Posed Variational Problems, R. Lucchetti and J. P. Revalski eds, Math. Appl., 331, Kluwer Academic, Dordrecht, Netherlands, 1995, 117-136.

22. K. Kimura, Y. C. Liou, S. Y. Wu and J. C. Yao, Well-posedness for parametric vector equilibbrium problems with applications, J. Indust. Manag. Optim., to appear.

23. A. S. Konsulova and J. P. Revalski, Constrained convex optimization problems well-posedness and stability, Numer. Funct. Anal. Optim., 15 (1994), 889-907.

24. B. Lemaire, C. Ould Ahmed Salem and J. P. Revalski, Well-posedness of variational problems with applications to staircase methods, C. R. Acad. Sci. Paris, 332, ser. 1 (2001), 943-948.

25. B. Lemaire, C. Ould Ahmed Salem and J. P. Revalski,

Well-posedness by perturbations of variational problems, J. Optim. Theory Appl., 115 (2002), 345-368.

26. E. S. Levitin and B. T. Polyak, Convergence of minimizing sequences in conditional extremum problems, Soviet Math. Dokl. 7 (1966), 764-767.

27. M. B. Lignola, Well-posedness and $L$-well-posedness for quasivariational inequalities, J. Optim. Theory Appl., 128 (2006), 119-138.

28. M. B. Lignola and J. Morgan, Well-posedness for optimization problems with constraints defined by variational inequalities having a unique solution, J. Glob. Optim., 16 (2000), 57-67.

29. M. B. Lignola and J. Morgan, $\alpha$-Well-posedness for Nash equilibria and for optimization problems with Nash equilibrium constraints, J. Glob. Optim., 36 (2006), 439-459.

30. R. Lucchetti and F. Patrone, A characterization of Tikhonov well-posedness for minimum problems, with applications to variational inequalities, Numer. Funct. Anal. Optim., 3 (1981), 461-476.

31. R. Lucchetti and F. Patrone, Hadamard and Tikhonov well-posedness of a certain class of convex functions, J. Math. Anal. Appl., 88 (1982), 204-215.

32. M. Margiocco, F. Patrone and L. Pusillo Chicco, A new approach to Tikhonov wellposedness for Nash equilibria, Optim., 40 (1997), 385-400.

33. J. Morgan and V. Scalzo, Discontinuous but well-posed optimization problems, SIAM J. Optim., 17 (2006), 861-870.

34. J. P. Revalski, Hadamard and strong well-posedness for convex programs, SIAM J. Optim., 7 (1997), 519-526. 
35. J. P. Revalski, Gereric properties concerning well-posed optimization problems, $C$. R. Acad. Bulgaria Sci., 38 (1985), 1431-1434.

36. A. N. Tikhonov, On the stability of the functional optimization problem, Soviet Comput. Math. Math. Phys., 6 (1966), 28-33.

37. T. Zolezzi, Well-posedness criteria in optimization with applications to the calculus of variations, Nonlinear Anal. TMA, 25 (1995), 437-453.

38. T. Zolezzi, Well-posedness and optimization under perturbations, Annals Oper. Res., 101 (2001), 351-361.

39. T. Zolezzi, On the distance theorem in quadratic optimization, J. Convex Anal., 9 (2002), 693-700.

40. T. Zolezzi, Condition number theorems in optimization, SIAM J. Optim., 14 (2003), 507-516.

41. T. Zolezzi, On well-posedness and conditioning in optimization, ZAMM Z. Angew. Math. Mech., 84 (2004), 435-443.

L. Q. Anh

Department of Mathematics,

Teacher College,

Cantho University,

Cantho City, Vietnam

E-mail: quocanh@ctu.edu.vn

P. Q. Khanh

Department of Mathematics,

International University of Hochiminh City,

Linh Trung, Thu Duc,

Hochiminh City, Vietnam

E-mail: pqkhanh@hcmiu.edu.vn

D. T. M. Van

Department of Mathematics,

Cantho College, Cantho City, Vietnam

E-mail: dangthimyvan@gmail.com

J.-C. Yao

Department of Applied Mathematics,

National Sun Yat-sen University,

Kaohsiung, Taiwan

E-mail: yaojc@math.nsysu.edu.tw 